\title{
Non Linear Image Restoration in Spatial Domain
}

\author{
Bushra Jalil, Fauvet Eric, Laligant Olivier
}

Le2i Laboratory, Universite de Bourgogne, Le Creusot, France.

Email: bushra.jalil@u-bourgogne.fr

Received May 10 ${ }^{\text {th }}, 2011$; revised June 22 ${ }^{\text {nd }}, 2011$; accepted July 30 ${ }^{\text {th }}, 2011$.

\begin{abstract}
In the present work, a novel image restoration method from noisy data samples is presented. The restoration was performed by using some heuristic approach utilizing data samples and smoothness criteria in spatial domain. Unlike most existing techniques, this approach does not require prior modelling of either the image or noise statistics. The proposed method works in an interactive mode to find the best compromise between the data (mean square error) and the smoothing criteria. The method has been compared with the shrinkage approach, Wiener filter and Non Local Means algorithm as well. Experimental results showed that the proposed method gives better signal to noise ratio as compared to the previously proposed denoising solutions. Furthermore, in addition to the white Gaussian noise, the effectiveness of the proposed technique has also been proved in the presence of multiplicative noise.
\end{abstract}

Keywords: Restoration, Nonlinear Filtering, Mean Square Error, Signal Smoothness

\section{Introduction}

The recovery of a signal from observed noisy data, while still preserving its important features, continues to remain a fundamentally elusive challenging problem in signal and image processing. More importantly, the need for an efficient image restoration method has grown with the massive production of digital images of different types. The two main limitations in any image accuracy are categorized as blur and noise. The main objective of any filtering method is to effectively suppress the noise elements.

Not only that, it is of extreme importance to preserve and enhance the edges at the same time. Several methods have been proposed in the past to attain these objectives and to recover the original (noise free) image. Most of these techniques uses averaging filter e.g. the Gaussian smoothing model has been used by Gabor [1], some of these techniques uses anisotropic filtering $[2,3]$ and the neighbourhood filtering [4,5] and some works in frequency domain e.g. Wiener filters [4]. In the past few years, wavelet transform has also been used as a significant tool to denoise the signal [6-8]. A brief survey of some of these approaches is given by Buades et al. [9]. Since the scope of this work is limited to spatial domain, therefore the proposed method has been compared with the classical methods for denoising in spatial domain.

Traditionally, linear models have been used to extract the noise elements e.g. Gaussian filter as they are com- putationally less expensive. However, in most of the cases the linear models are not able to preserve sharp edges which are later recognized as discontinuities in the image. On the other hand, nonlinear models can effectively handle this task (preserve edges) but more often, non linear model are computationally expensive. In the present work, we attempt to propose a non linear model with the very less computational cost to restore image from noisy data samples. The method utilizes data samples and find the best compromise between the data samples and smoothness criteria which ultimately result in giving the denoise signal at a very low computational cost. We have also presented the comparative analysis of the present technique with some of the previously proposed method.

The paper is organized as follows. The principle of the proposed technique is given in Section 2. Section 3 explains the overview of the restoration method. Application on images and comparative analysis is given in Section 4 and finally Section 5 concludes the work with some future perspectives.

\section{Principle of the Method}

We assume that the given data specify the model:

$$
y_{i j}=f\left(x_{i j}\right)+\epsilon_{i j} \text { where } i, j=1, \cdots, n
$$

$f$ is the noise free signal, uniformly sampled (e.g., an image) and $\epsilon_{i j}$ is the white Gaussian noise $N\left(0, \sigma^{2}\right)$. 
In the present work, the given data will always be an $n \times n$ matrix with $n=2^{N}$. The aim of the current work is to estimate the function $F=\left(f\left(x_{i j}\right)\right)_{i, j=1}^{n}$ with respect to an estimator $\ddot{F}_{i, j}$ such that:

$$
\operatorname{SNR}(d B)=-10 \log 10 \frac{\sum_{i, j=1}^{n}\left(\ddot{F}_{i, j}-F_{i, j}\right)^{2}}{\sum_{i, j=1}^{n} F_{i, j}{ }^{2}}
$$

In order to estimate the function $F$, the method utilizes the data samples and performs non linear functioning to estimate the best fit. The filtering has been performed on each row and column matrix individually and the final stage by utilizing filtering in $x$ and $y$ directions yield in fully denoised image.

$$
G=\frac{1}{\sqrt{2}} \sqrt{\left(G_{x}\right)^{2}+\left(G_{y}\right)^{2}}
$$

where $1 / \sqrt{2}$ the normalizing is factor, $G_{x}$ is the filtered image in horizontal direction and $G_{y}$ is the filtered image in vertical direction.

\section{Restoration Method}

In this section, a brief explanation of the proposed denoising method (Mse-Smooth) is given. The restoration method utilizes all sampled points and smoothness of the signal to estimate the best fit by working in an iterative mode.

The method is design for one dimensional signal; therefore it performs non linear filtering on image initially row by row and then column by column. It means if the size of the image is $n \times n$, then there would be $2 n$ one dimensional signals (correspond to the operation in horizontal and vertical direction). At the final stage, by using expression in given Equation (3) merging of filtering operation in horizontal and vertical direction results in giving the fully denoised image.

\section{Denoising Method}

As explained before, in the present work filtering operation is performed on each row and column individually. Therefore let's define any one dimensional signal as:

$$
Y^{k+1}=Y^{k}+\lambda^{k}\left(-\frac{\partial C^{M S E, k}}{\partial Y}\right)+\gamma^{k}\left(-\frac{\partial C^{M S O, k}}{\partial Y}\right)
$$

$Y$ denotes the one dimensional signal with $l \in 1, \cdots, 2^{N}$ samples. $K$ defines the iteration step. $C^{M S E}$ is the mean square error estimation of the restored subset with the original signal $\left(Y_{o}\right)$ such that:

$$
C^{M S E}=\sum_{l=1}^{N}\left(y_{l}{ }^{k}-y_{o}{ }^{k}\right)
$$

$C^{M S O}$ defines the smoothness of the reconstructed subset signal.

$$
C^{M S E}=\sum_{l=1}^{N} y_{l}^{k^{\prime \prime 2}}
$$

In order to find the value of $\lambda^{\mathrm{k}}$ and $\gamma^{\mathrm{k}}$ in Equation (4), consider the Taylor series expansion, and for the given series, to find the minimum mean square error we want $f^{\prime}(x+d x)=0$; therefore, by simplifying the Taylor series expansion,

$$
\mathrm{d} x=-\frac{f^{\prime}(x)}{\frac{d^{2} f}{\mathrm{~d} x^{2}}}
$$

and we know that

$$
x^{k+1}=x^{k}+\lambda d x
$$

As $\quad x \rightarrow y^{k+1}$ and $f \rightarrow C^{M S E, k}$ the variables in Equation (8) can be replaced such that

$$
Y^{k+1}=Y^{k}+\frac{-C^{M S E, k}}{\frac{d^{2} C^{M S E, k}}{d Y^{k^{2}}}}
$$

Thus,

$$
\lambda^{k}=\frac{\mathrm{d}^{2} C^{M S E, k}}{\mathrm{~d} Y^{k^{\wedge}}}
$$

and we can simplify the equation to

$$
Y^{k+1}=Y^{k}+\lambda^{k}\left(-\frac{\left(\partial C^{M S E}\right)}{\partial Y}\right)
$$

Similarly, by using the Taylor series as smoothing criteria, $\gamma^{k}$ is as follows

$$
\gamma^{k}=\frac{\mathrm{d}^{2} C^{M S O, k}}{\mathrm{~d} Y^{k^{2}}}
$$

By using Equation (4), each row wise and column wise vector were restored individually and result in giving two $n \times n$ matrices, $G_{x}$ and $G_{y}$ respectively. At the last stage, by using mathematical expression given in Equation (3), the final denoised image has been restored.

\section{Results and Discussions}

The test image use in this work is Lena $256 \times 256$ picture. We generated noisy data from clean image by adding pseudorandom numbers (white gaussian noise) resulting in signal to noise ration (SNR) of approximately $15 \mathrm{~dB}$ (SNR is defined in Equation (2)). Figure 1 shows the corrupted Lena image with white Gaussian noise (15 dB) and the denoising result with the proposed technique as shown in Figure 2(f). It can be seen from the figure that, the new method denoised the image reasonably well 
while keeping the edges preserved.

In order to illustrate the amount of the noise in the data and the effects of the denoising, we show in Figure 3, a single row (100) of the image (15 dB white Gaussian noise), plotted as a curve. It can be seen from the figure that the method not only smooth the noisy part of the signal but also preserve the sharp edges or transitions to good extent. At the same time, the proposed method performs the whole operations at a very low computa-

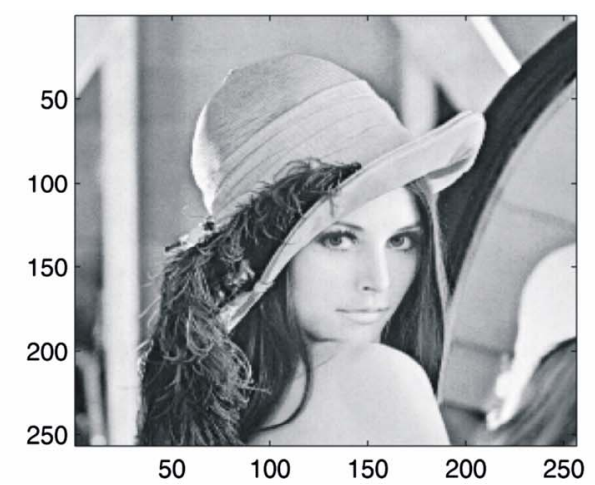

(a) tional cost which makes it useful for many real time applications (maximum time taken for any experiment was approx $16 \mathrm{sec}$ ).

\subsection{Comparative Analysis}

The human eye is able to decide if the quality of the image has been improved by the denoising method. Figure 2 displays the comparative analysis of the proposed method with other smoothing filters including Non-Locals

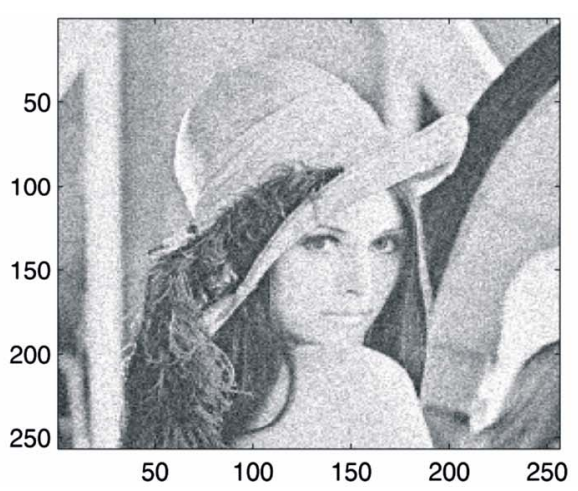

(b)

Figure 1. (a) Original Lena image, (b) Lena image with "white Gaussian noise” SNR of 15 dB.

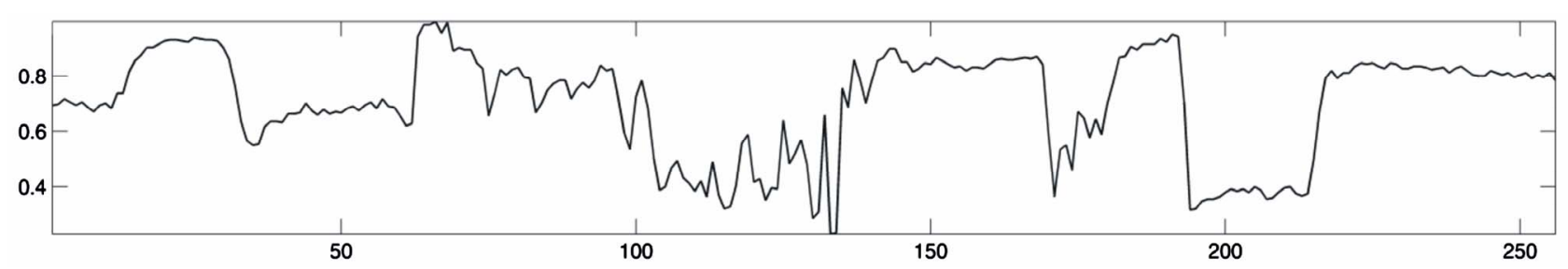

(a)

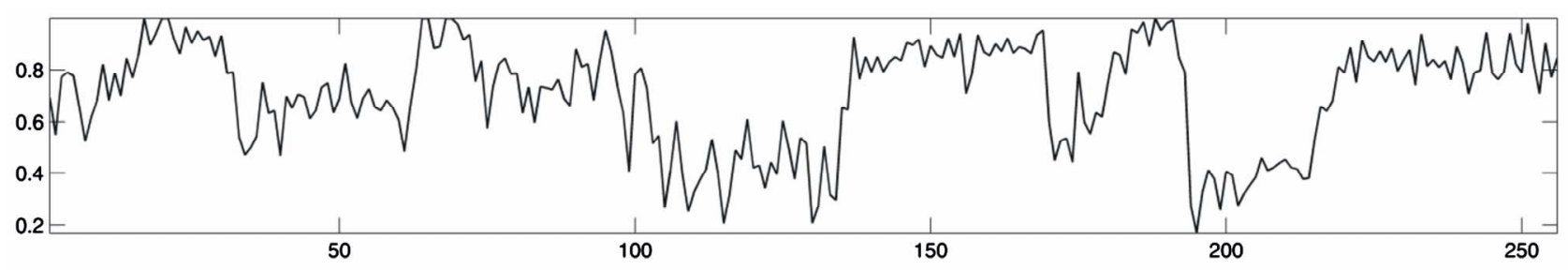

(b)

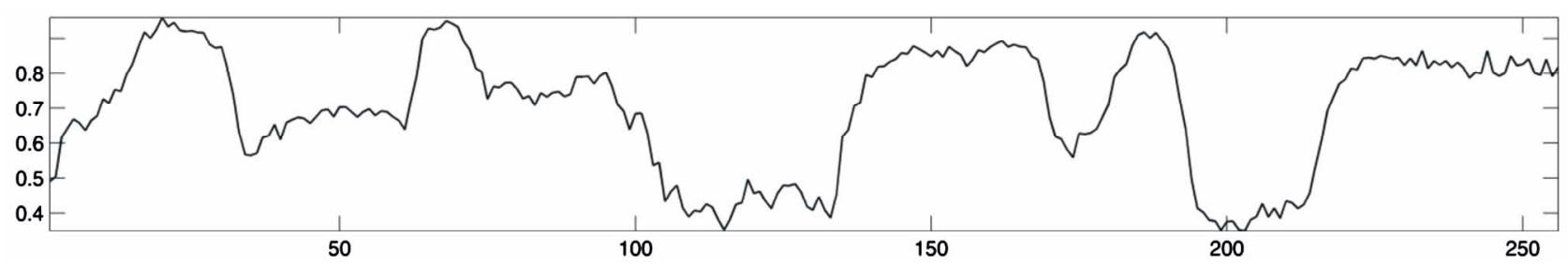

(c)

Figure 2. (a) Original Lena image, (b) Lena image with “white Gaussian noise” SNR of 15 dB, (c) Denoised Lena image with Visu shrink method, (d) Denoising with Wiener filtering, e) Denoising with Non Local means, f) Denoising with proposed Mse-Smooth method. 


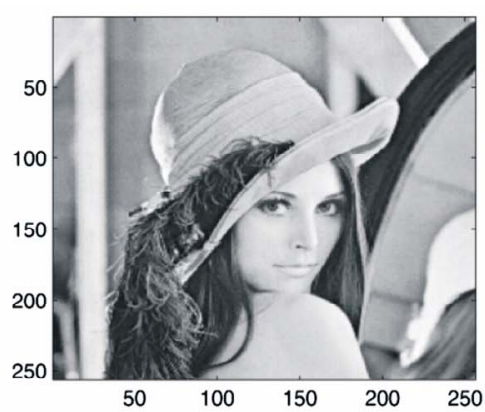

(a)

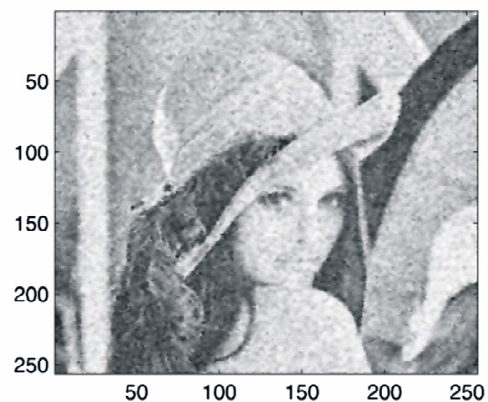

(d)

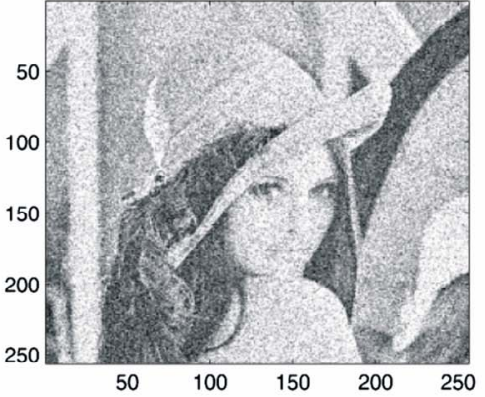

(b)

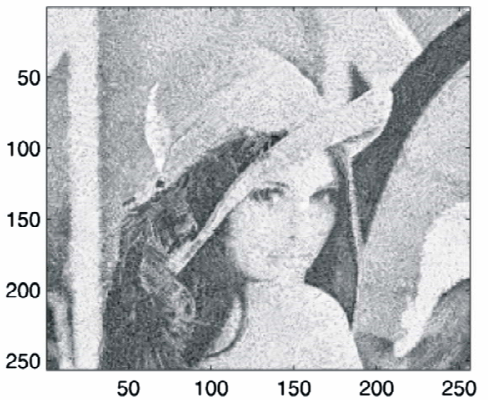

(e)

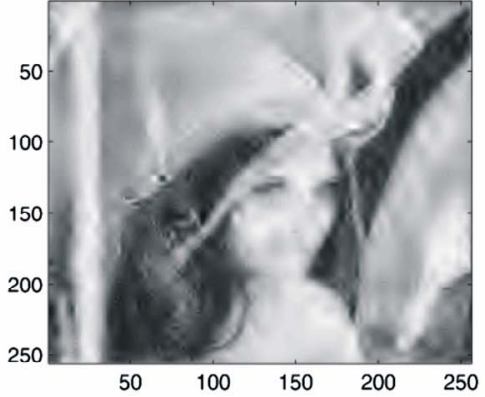

(c)

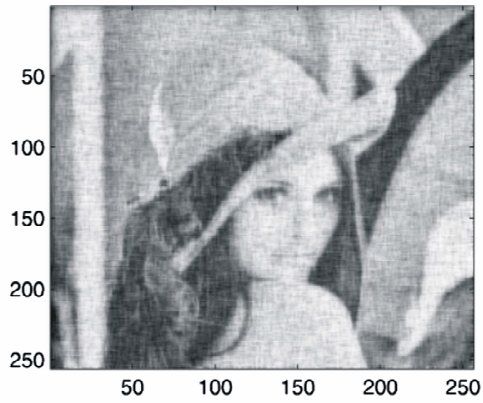

(f)

Figure 3. (a) Row 100 of original Lena image, (b) Row 100 of noisy Lena image with "white Gaussian noise” SNR of $15 \mathrm{~dB}$, c) Row 100 of denoised Lena image with proposed Mse-Smooth method.

[10], Wiener filter [4] and classical VISU shrink method [7]. The experiment has been simulated by adding white Gaussian noise. It can be seen from the figure that the VISU shrink results in giving good smoothness but at the cost of the edges. Not a single edge has been preserved; this however is not, in the case of NL-means or Wiener filtering. NL-means and Wiener filtering preserved the edges reasonably well, but in this case the noise elements are visible (clearly visible on the background of Lena) and can be seen with the naked eye as well. The proposed (Mse-Smooth) method gave the best compromise of the two (blur and noise artefacts) as shown in Figure 2.

\subsection{Test with Multiplicative Noise}

At the second stage, the method has been tested with the addition of multiplicative noise. In the case of multiplicative noise, variance of the noise is a function of the signal amplitude. Figure 4 shows a comparison between one dimensional step like signal corrupted with an additive white Gaussian noise and multiplicative noise. In the case of multiplicative noise, the noise variance is higher when amplitude of the signal is higher as shown in Figure 4. In relation to the images, noise in bright regions have higher variations and could be interpreted wrongly as features in the original image [11]. Thus, it is harder and more complicated to smooth the noise without de- grading true image features.

The proposed method has been tested with an addition of multiplicative noise as well. Figure 5 illustrates the amount of the multiplicative (speckle $15 \mathrm{~dB}$ ) noise in the data samples and the effects of the denoising with the proposed method, a single row (100) of the image with $15 \mathrm{~dB}$ speckle noise, plotted as a curve. It can be seen from the figure that in this case as well, the method smooth the noisy part of the signal reasonably well and also sharp edges are preserved. Figure 6 illustrate the results on real Lena image. It can be seen from the figure that the proposed method gave the best performance in the presence of multiplicative noise. Table 1 summarizes the numerical results in term of SNR (dB) of the new method and some of the previously proposed techniques with different types of noise. We can see that the new method out performs VISU shrink, NL means and Wiener filtering techniques in different cases.

\section{Conclusions}

In this work, we have presented a new denoising algorithm, based on the actual noisy image samples. Unlike other existing techniques, we have not considered modelling of an image or noise characteristics in developing the approach. Instead we have estimated the best fit of the signal by utilizing actual noisy data samples and smoothness criteria. At the same time, the proposed non 


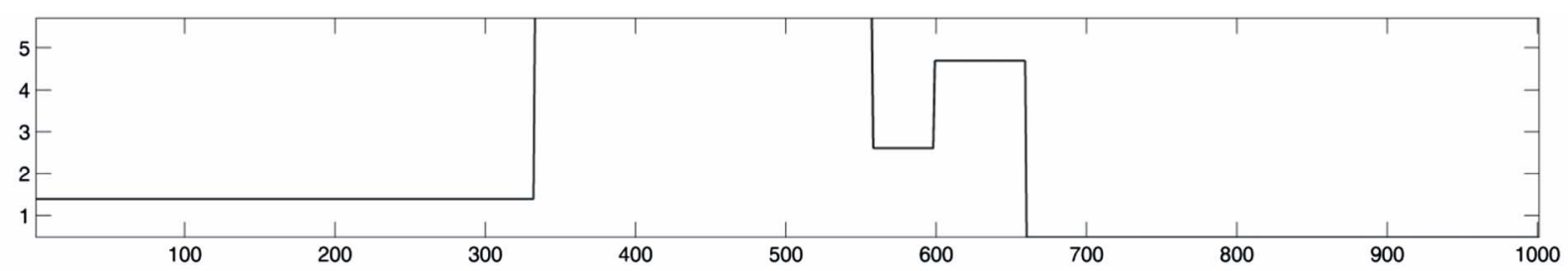

(a)

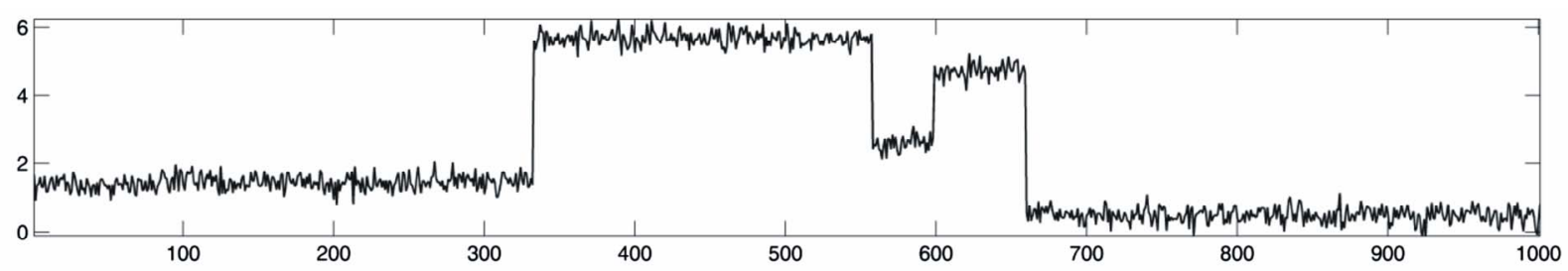

(b)

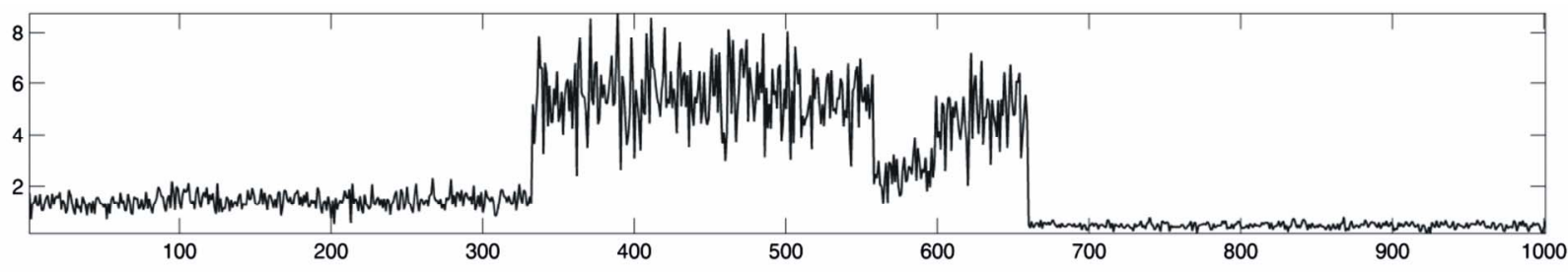

(c)

Figure 4. (a) Synthetic “Step signal”, (b) Step signal with the addition of white Gaussian noise 15dB, (c) Step signal with an addition of Speckle (multiplicative noise) $15 \mathrm{~dB}$.

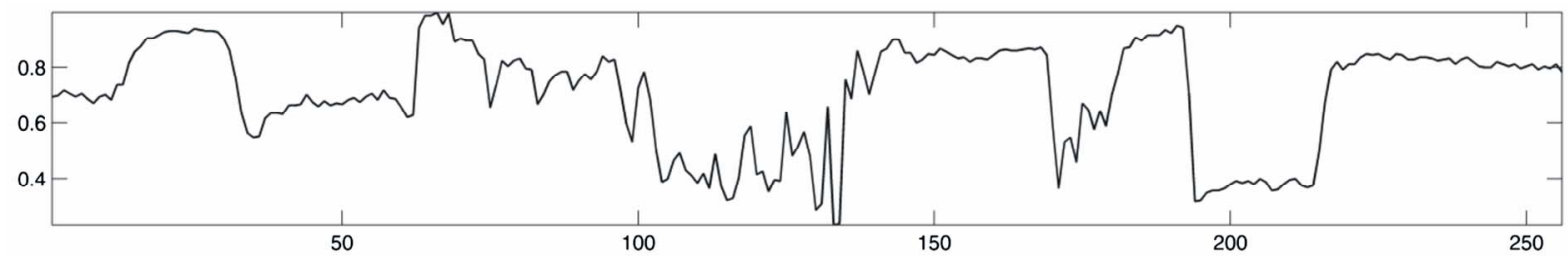

(a)

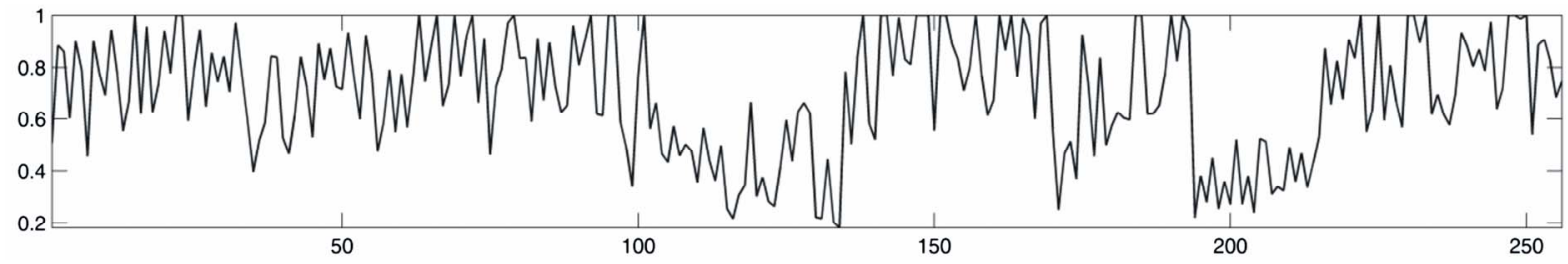

(b)

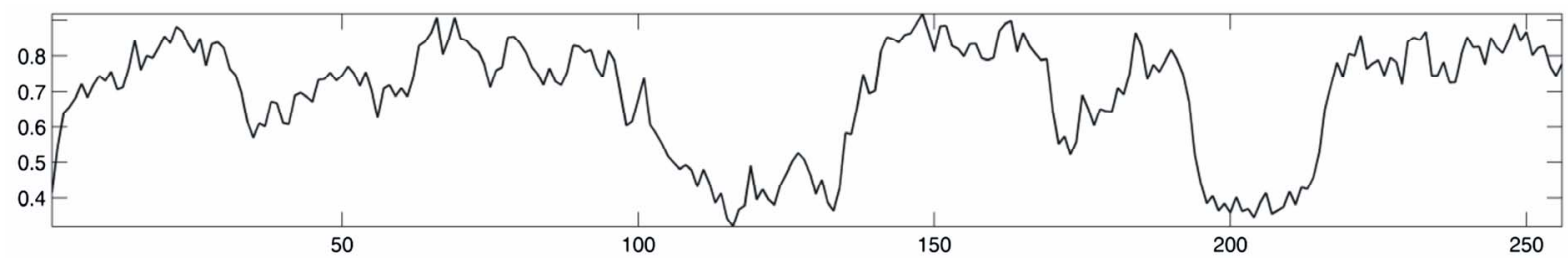

(c)

Figure 5. (a) Row 100 of original Lena image, (b) Row 100 of noisy Lena image corrupted with “Speckle noise” SNR of 15dB, (c) Row 100 of denoised Lena image with proposed Mse-Smooth method. 


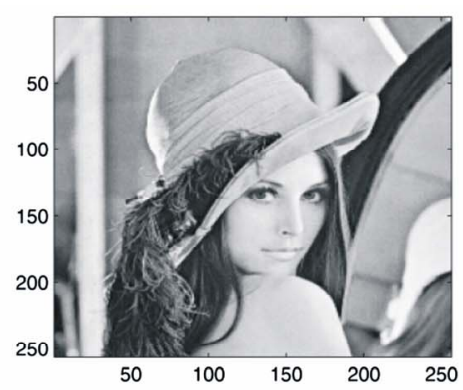

(a)

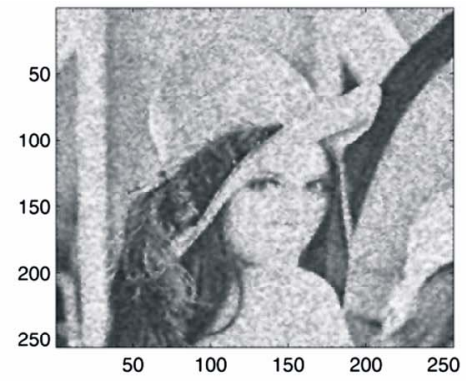

(d)

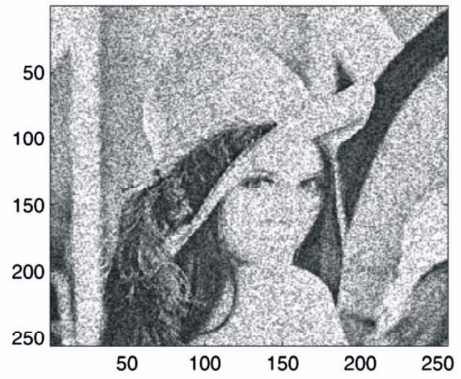

(b)

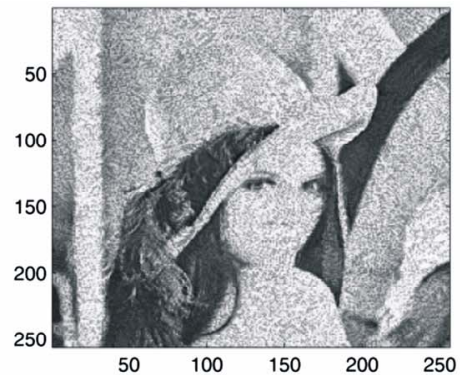

(e)

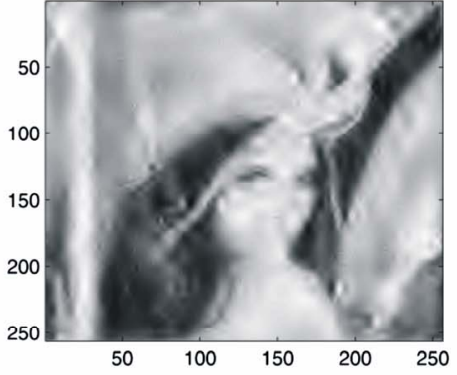

(c)

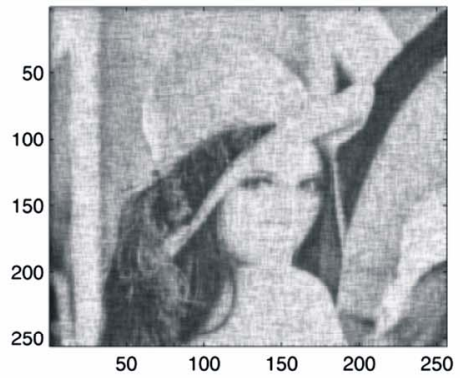

(f)

Figure 6. (a) Original Lena image, (b) Lena image with "Speckle noise” SNR of 15 dB, (c) Denoised Lena image with Visu shrink method, (d) Denoising with Wiener filtering, (e) Denoising with Non Local mean, (f) Denoising with proposed MseSmooth method.

Table 1. Statistical Results in terms of SNR (dB) of "Lena Image” with different types of noise.

\begin{tabular}{llllll}
\hline Noise Type & Input & NLM & Weiner & VISU Shrinkage & Mse-Smooth \\
\hline Gaussian & 15.02 & 18.28 & 21.49 & 19.44 & 21.23 \\
Speckle & 17.55 & 18.75 & 23.76 & 20.73 & 22.32 \\
Salt/Pepper & 15.10 & 15.90 & 17.23 & 16.60 & 20.47 \\
\hline
\end{tabular}

linear image filtering technique works equally well in the presence of signal dependent nature of multiplicative noise in spatial domain. The proposed non linear expression has effectively directed the algorithm in the smoothing operation at a very low computational cost. For images, it is important that edges data should be preserved. The denoising algorithm presented in this work, to a large extent has satisfied the constraint that phase should not be corrupted. The effectiveness of this technique encourages the possibility of improving this approach to preserve the edges to more extent.

\section{REFERENCES}

[1] A. Bruckstein M. Lindenbaum and M. Fischer, "On Gabor Contribution to Image Enhancement. Pattern recognition,” Computer Methods and Programs in Biomedicine, Vol. 27, No. 1, 1994, pp. 1-8.

[2] J. Malik and P. Perona, "Scale Space and Edge Detection Using Anisotropic Diffusion,” IEEE Transactions on Pat- tern Analysis, Vol. 12, No. 7, March 1990, pp. 629-639.

[3] J. M. Morel L. Alvarez and P. L. Lions, "Image Selective Smoothing and Edge Detection by Nonlinear Diffusion," Journal of Numerical Analysis, Vol. 29, No. 1, 1992, pp. 182-193.

[4] L. Yaroslavsky, "Digital Picture Processing-An Introduction,” Springer-Verlag, Berlin, 1985.

[5] R. Manduchi and C. Tomasi, "Bilateral Filtering for Gray and Color Images," Proceedings of the Sixth International Conference on Computer Vision, Bombay, 4-7 January 1998, pp. 839-846.

[6] D. Donoho and R. Coifman, "Wavelets and Statistics, Chapter Translation Invariant Denoising," Springer-Verlag, Berlin, 1995, pp. 125-150.

[7] D. Donoho, “Denoising by Soft-Thresholding," IEEE Transactions on Information Theory, Vol. 41, 1995, pp. 613-627.

[8] A. Alecu, A. Munteanu, L. Tessens and A. Pizurica, "Context Adaptive Image Denoising through Modeling of Curvelet Domain Statistics,” Journal of Electronic Imag- 
ing, Vol. 17, No. 3, 2008, p. 033021. doi:10.1117/1.2987723

[9] J. Morel, A. Buades and B. Coll, "On Image Denoising Methods,” Technical Report CMLA, 2004.

[10] J. M. Morel, A. Buades and B. Coll, "A Non-Local Algorithm for Image Denoising,” IEEE Computer Society
Conference on Computer Vision and Pattern Recognition, San Diego, 20-26 June 2005, pp. 60-65.

[11] A. Reza and Y. Hawwar, "Spatially Adaptive Multiplicative Noise Image Denoising Technique,” IEEE Transactions on Image Processing, Vol. 11, No. 12, 2002, pp. 1397-1404. doi:10.1109/TIP.2002.804526 